\title{
Similaridade da fauna de Chiroptera (Mammalia), da Serra Negra, municípios de Rio Preto e Santa Bárbara do Monte Verde, Minas Gerais, com outras localidades da Mata Atlântica
}

\author{
Pedro Henrique Nobre ${ }^{1,5}$, Alexmar dos Santos Rodrigues ${ }^{2}$, Igor de Almeida Costa ${ }^{3}$, \\ Artur Emílio da Silva Moreira ${ }^{4}$ \& Hugo Henrique Moreira ${ }^{4}$ \\ ${ }^{1}$ Departamento de Ciências Naturais, Colégio de Aplicação João XXIII, \\ Universidade Federal de Juiz de Fora - UFJF, \\ Rua Visconde de Mauá, 300, Santa Helena, CEP 36015-260, Juiz de Fora, MG, Brasil \\ ${ }^{2}$ Programa de Iniciação Científica FAPEMIG, Universidade Federal de Juiz de Fora - UFJF, \\ ${ }^{3}$ Centro de Ensino Superior de Juiz de Fora - CESJF \\ ${ }^{4}$ Programa de Iniciação Científica, Universidade Federal de Juiz de Fora - UFJF, \\ ${ }^{5}$ Autor para correspondência: Pedro Henrique Nobre, e-mail: pedro.nobre@ufjf.edu.br
}

NOBRE, P.H., RODRIGUES, AS., C.I.A., MOREIRA, A.E.S. \& MOREIRA, H.H. Similarity of the bat fauna (Mammalia) in Serra Negra, Rio Preto and Santa Bárbara do Monte Verde municipalities, Minas Gerais, with other localities of Atlantic Forest. Biota Neotrop. 9(3): http://www.biotaneotropica.org.br/v9n3/en/ abstract?article+bn03309032009.

\begin{abstract}
The southern slope of the Serra Negra region is located between the Rio Preto and Santa Bárbara do Monte Verde municipalities, state of Minas Gerais. The region is a mountain chain inserted in the Atlantic rainforest domain. The studies were conducted in a rainforest at elevations of 800-1,200 m, where bats were captured in mist-nets installed at the ground level in the interior and edges of the forest. After 14 monthly samplings and total capture effort of 22,140 $\mathrm{m}^{2}$ r.h, 246 bats belonging to Phyllostomidae (12 species) and Vespertilionidae ( 3 species) were captured. Sturnira lilium was the most frequently captured species, followed by Carollia perspicillata, Desmodus rotundus and Artibeus lituratus (33.7,21.1, 19.1 and 16,3\% of the captures, respectively). Cluster Analysis including Serra Negra and bat checklists of other patches of Atlantic forest revealed that Serra Negra is more similar to the Serra dos Órgãos, State of Rio de Janeiro, southeastern Brazil, than the other areas.
\end{abstract}

Keywords: Atlantic forest, bat diversity, highland forest, southeastern Brazil.

NOBRE, P.H., RODRIGUES, AS., C.I.A., MOREIRA, A.E.S. \& MOREIRA, H.H. Similaridade da fauna de Chiroptera (Mammalia) da Serra Negra, municípios de Rio Preto e Santa Bárbara do Monte Verde, MG, com outras localidades da Mata Atlântica. Biota Neotrop. 9(3): http://www.biotaneotropica.org.br/v9n3/en/ abstract?article+bn03309032009.

Resumo: A face sul da região da Serra Negra está localizada entre os municípios de Rio Preto e Santa Bárbara do Monte Verde no estado de Minas Gerais. A região é marcada por um relevo montanhoso e vegetação formada por Floresta Ombrófila Densa. Os estudos foram conduzidos em altitudes entre 800 a 1.200 m onde os morcegos foram capturados em redes de neblina instaladas principalmente no interior e borda de mata. Durante um período de 14 meses e um esforço de captura de $22.140 \mathrm{~m}^{2}$ r.h, foram capturados 246 morcegos pertencentes a 15 espécies, 13 gêneros e 2 famílias: Phyllostomidae (12 espécies) e Vespertilionidae (3 espécies). A espécie Sturnira lilium foi a mais freqüente com 33,7\% das capturas, seguida por Carollia perspicillata com 21,1\%, Desmodus rotundus com $19,1 \%$ e Artibeus lituratus com 16,3\%. Em análise de agrupamento realizada entre a fauna de Chiroptera da face sul da Serra Negra com outros levantamentos, a área de estudo se mostrou similar a fauna de morcegos do Parque Nacional da Serra dos Órgãos.

Palavras-chave: diversidade, morcegos, Floresta Atlântica, Floresta de altitude. 


\section{Introdução}

O Brasil abriga uma das maiores diversidades de mamíferos, com 652 espécies descritas (Reis et al. 2006). A ordem Chiroptera é a segunda em número de espécies entre os mamíferos, superada apenas pelos roedores (Rodentia). No Brasil, os morcegos estão representados por nove famílias, 64 gêneros e 167 espécies (Reis et al. 2007a), representados apenas pela subordem Microchiroptera.

Minas Gerais abriga três dos biomas mais importantes do Brasil, sendo dois considerados hotspots (Mata Atlântica e Cerrado), ou seja, regiões com uma rica biodiversidade e um alto grau de ameaça (Mittermeier et al., 2005). A amostragem existente até o momento é extremamente baixa e insuficiente para se conhecer a diversidade de quirópteros. Esbérard \& Bergallo (2003), analisaram os inventários realizados nos estados do Rio de Janeiro, São Paulo e Minas Gerais com o objetivo de se obter um perfil dos estudos desenvolvidos com morcegos. Os resultados obtidos demonstraram a grande diferença entre os estados em relação ao número de capturas. O estado do Rio de Janeiro apresentou 70,16\% das capturas, São Paulo com 26,68\% e Minas Gerais com apenas $6,6 \%$; o que revela a grande deficiência de inventários neste último.

A região da Serra Negra está inserida no complexo Serra da Mantiqueira e no Corredor Ecológico da Mantiqueira, sendo também considerada como área de prioridade para conservação da biodiversidade no Brasil (Drummond et al. 2005). Neste sentido o estudo teve como objetivo conhecer a diversidade da fauna de morcegos da Serra Negra e Serra do Funil, entre os municípios de Rio Preto e Santa Bárbara do Monte Verde, Zona da Mata de Minas Gerais.

\section{Material e Métodos}

\section{1. Área de estudo}

A Serra Negra possui uma extensão de aproximadamente $15 \mathrm{~km}$ limitada a norte pelo município de Lima Duarte, $21^{\circ} 58^{\prime} 11^{\prime \prime} \mathrm{S}$ e $43^{\circ} 53^{\prime} 21^{\prime \prime} \mathrm{O}$; ao sul pelo município de Rio Preto, $22^{\circ} 01$ ' $46,4^{\prime \prime} \mathrm{S}$ e $43^{\circ}$ 52' 31,5"; a leste pelo município de Santa Bárbara do Monte Verde, $21^{\circ} 58^{\prime} 21^{\prime \prime} \mathrm{S}$ e $43^{\circ} 50^{\prime} 06^{\prime \prime}$ e a oeste pelo município de Bom Jardim de Minas, $21^{\circ} 58^{\prime} 53^{\prime \prime} \mathrm{S}$ e $43^{\circ} 56^{\prime} 08^{\prime \prime} \mathrm{O}$. O gradiente altitudinal encontrado na Serra Negra varia entre 900 e 1.760 m, sendo as cotas mais baixas da região situadas junto ao leito à jusante do Rio Preto, entre 400-500 m de altitude (Heilbron et al. 2000). O clima é do tipo Cwb (Köppen), mesotérmico úmido com precipitação média de $1946 \mathrm{~mm}$.

O complexo de serras da Mantiqueira, do qual a Serra Negra faz parte, apresenta formações florestais que variam desde Floresta Estacional Semidecidual Baixo-Montana, Florestas Ombrófilas Baixo-Montana e Alto-Montana (Oliveira-Filho \& Fontes 2000) a Floresta Ombrófila Mista (Veloso et al. 1991). Essas florestas se encontram nos estágios variados de desenvolvimento sucessional, associada aos campos de altitude ou aos campos rupestres, apresentando diversos microhábitats e formações ecotonais diversas (Benites et al. 2003).

A Serra Negra está incluída na região de Bom Jardim, com alta prioridade para conservação em Minas Gerais, sendo recomendada investigações científicas em forma de inventários devido à alta diversidade e baixo conhecimento científico da área (Drummond et al. 2005). A Serra Negra faz parte do Corredor Ecológico da Mantiqueira (Costa \& Herrmann 2006) e do Núcleo Ibitipoca, formado por seis municípios com apenas uma Unidade de Conservação de Proteção Integral Estadual, o Parque Estadual do Ibitipoca.

Para este estudo foram realizadas capturas de morcegos em fragmentos florestais localizados na vertente sul da Serra Negra, entre os municípios de Rio Preto e Santa Bárbara do Monte Verde, com variação de altitude entre 800 a $1.200 \mathrm{~m}$.

\section{Captura e coleta dos morcegos}

As campanhas de campo foram realizadas mensalmente de novembro de 2007 a novembro de 2008, com pelo menos duas noites de captura por campanha. Foram utilizadas redes de neblina de $9 \times 2,5 \mathrm{~m}$ e $6 \times 2,5 \mathrm{~m}$ armadas a partir do solo e abertas no crepúsculo. Foram instaladas aproximadamente 10 redes por noite, permanecendo abertas por 6 horas, totalizando $22.140 \mathrm{~m}^{2}$ r.h de esforço de captura. Os pontos de instalação das redes foram escolhidos de acordo com os seguintes critérios: presença de possíveis abrigos naturais, bordas de mata, clareiras, matas ciliares, próximo à cursos d'água, junto a árvores em frutificação ou potenciais abrigos noturnos e outros que apresentaram condições favoráveis à captura de morcegos.

Durante os trabalhos de captura, as redes foram vistoriadas a cada 15 minutos e os morcegos capturados foram mantidos temporariamente em sacos de tecido de algodão individuais, até o momento de triagem e identificação. Após o horário de captura as redes foram fechadas e os morcegos soltos neste mesmo local.

Com o objetivo de se manter uma coleção de referência para a diversidade da região, 28 espécimes foram tombados como material testemunho (Apêndice 1). Os morcegos coletados foram sacrificados e fixados em formol a $10 \%$, conservados em álcool $70^{\circ} \mathrm{gl}$, de acordo com Vizotto \& Taddei (1973), sendo devidamente incluídos na coleção de Chiroptera da Universidade Federal de Juiz de Fora. A identificação das espécies seguiu os critérios de Vizotto \& Taddei (1973); Barquez et al. (1993); Simmons \& Voss (1998); Medellin et al. (1997), Reis et al. (2007b) e Redondo et al. (2008) para o gênero Artibeus.

Os trabalhos de captura e coleta de morcegos foram conduzidos de acordo com a autorização para atividades com finalidade científica, Número: 10767-1, expedida pelo Instituto Brasileiro do Meio Ambiente e dos Recursos Naturais Renováveis - IBAMA.

\section{Análise dos dados}

Para calcular o esforço de captura com a utilização de redes de neblina, foi utilizado a metodologia proposta por Straube \& Bianconi (2002), onde multiplica-se o somatório de metros quadrados de redes pelo número de horas de permanência das redes abertas. Para comparação da diversidade de morcegos encontrada na região da Serra Negra com outras localidades, foram selecionados 12 levantamentos em áreas sob o domínio da Mata Atlântica. A escolha das localidades se deu em função da disponibilidade de informações, número de capturas obtido (equivalente ou próximo ao da região da Serra Negra), metodologia adotada e semelhança entre gradientes altitudinais. A análise dos dados foi obtida através do programa estatístico MVSP 3.1, com distância euclideana e utilizando-se o método de agrupamento não ponderado - UPGMA.

\section{Resultados e Discussão}

Durante um período de 13 meses e um esforço de captura de $22.140 \mathrm{~m}^{2}$ r.h, foram capturados 246 indivíduos pertencentes a 15 espécies, 13 gêneros e 2 famílias (Apêndice 2). A família Phyllostomidae foi a mais abundante atingindo $97,6 \%$ das capturas e quase a totalidade das espécies registradas. A família Vespertilionidae foi representada apenas por seis capturas e três espécies. Entre os Phyllostomidae, S. lilium foi a espécie mais freqüente com $33,6 \%$ das capturas, seguida por $C$. perspicillata com $21,1 \%, D$. rotundus com $19 \%$ e quarto mais freqüente foi Artibeus lituratus com 15,8\%. As quatro espécies juntam somam $89,5 \%$ das capturas e as 11 espécies restantes representam apenas $10,5 \%$ de todos os registros. Com exceção de um espécime 
de Myotis nigricans coletado em habitação humana com puçá, todos os registros foram obtidos em redes de neblina.

A predominância da família Phyllostomidae pode estar relacionada à metodologia de captura empregada. $\mathrm{O}$ uso de redes de neblina instaladas no sub-bosque privilegia a captura deste grupo (Simmons \& Voss, 1998), visto que a maioria destes animais utiliza o subosque na procura de alimento. Além disso, a família Phyllostomidae é a mais diversa entre os morcegos da região Neotropical (Fenton et al. 1992), correspondendo a respectivamente $55 \%$ das espécies registradas no Brasil (Peracchi et al., 2006).

Algumas espécies capturadas na Serra Negra podem ser consideradas de ocorrência mais rara que as demais, e merecem destaque por serem registradas já na fase inicial de capturas. Chrotopterus auritus, representada por 3 capturas, é pouco registrada em inventários com baixo número de capturas; Mimom bennettii, juntamente com Micronycteris minuta, apesar de serem consideradas espécies de baixo risco à extinção, apresentam ocorrência sempre baixa em estudos de diversidade e abundância de Chiroptera (Apêndice 2).

Comparando a composição da fauna de Chiroptera na região da Serra Negra com outras localidades, podemos considerar dois agrupamentos de importância: o agrupamento 1 formado pela Serra Negra e Serra dos Órgãos, que apresenta a menor distância euclidiana dentre todos os outros agrupamentos obtidos; e o agrupamento 2, formado pela Serra do Ibitipoca e Serra do Caraça (Figura 1).

O Parque Nacional Serra dos Órgãos faz parte do Bloco da Região Serrana Central do estado do Rio de Janeiro, segundo a classificação de Rocha et al. (2003), cuja vegetação predominante é a Floresta Ombrófila Densa e nas áreas com altitudes entre 500 a 1.500 m surge a Floresta Ombrófila Densa Montana. Nesta região as chuvas são bem distribuídas e a precipitação média é de $1.500 \mathrm{~mm}$.

Na Serra Negra a cobertura vegetal é representada pela Floresta Ombrófila Densa (Veloso et al., 1991; Valente, 2007) e pelos campos rupestres. A precipitação média anual é de 1.886 mm (Valente, 2007), com gradiente altitudinal entre 900 e $1.760 \mathrm{~m}$. As duas localidades apresentam semelhanças quanto aos gradientes altitudinais e precipitações médias anuais. Podemos considerar também a face sul da região da Serra Negra, como uma área que sofre forte influência da umidade, como pode ser constatado também na Serra dos Órgãos. É possível então que o ambiente formado pelo clima, tipo de vegetação e altitude foi responsáveis por este agrupamento.

A Serra Negra apresentou similaridade também com a região da Serra das Almas localizada no estado do Ceará. Esta região é formada por ambientes de Mata Atlântica (floresta decidual) em altitude superior a $750 \mathrm{~m}$, carrasco e caatinga em altitudes inferiores a 700 m (Silva et al. 2004). A presença de Mata Atlântica formando um mosaico com outros tipos de ambientes ocorre também na Serra Negra, sendo que estas características em comum podem ser uma hipótese para explicar a inclusão da Serra das Almas no agrupamento formado pela Serra Negra e Serra dos Órgãos.

O Parque Estadual do Ibitipoca e a Serra do Caraça, também formaram um agrupamento (Agrupamento 2, Figura 1) e apresentam características similares quanto à vegetação, presença marcante de campos rupestres, altitudes semelhantes e presença de grandes cavernas em quartzito. Em ambas as localidades, Sturnira lilium foi muito abundante, característica observada também na Serra Negra. $\mathrm{O}$ alto índice de capturas de $S$. lilium pode ser comparado com outras áreas de altitude semelhante, como a Serra do Caraça, apresentado por Falcão et al. (2003), onde observa-se a ocorrência de $64 \%$ de S. lilium, num total de 246 espécimes capturados, distribuídos em 15 espécies.

A partir de várias análises geradas por este estudo observou-se que, quando não são incluídos os dados referentes a Serra dos Órgãos, a Serra Negra agrupa-se com o Parque Estadual do Ibitipoca. Além

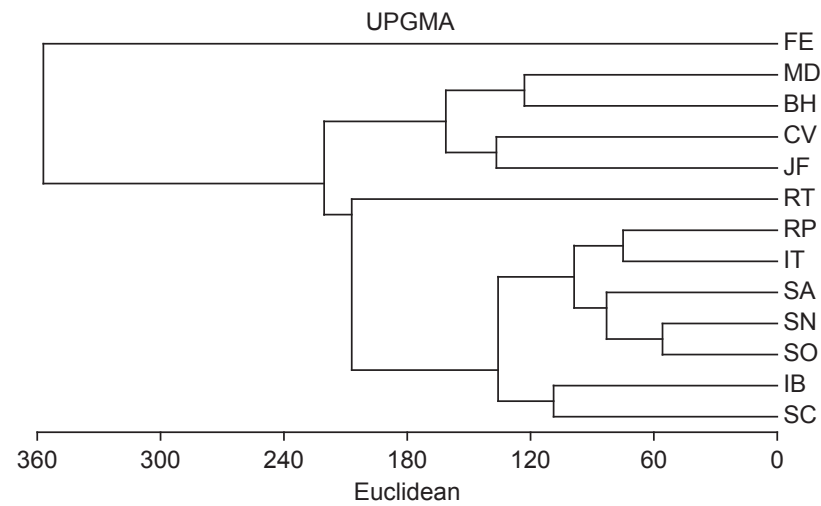

Figura 1. Dendograma com distância euclideana gerado pelo método de UPGMA para 13 levantamentos de Chiroptera incluindo a região da Serra Negra e as seguintes localidades e estados: FE- Município de Fênix - PR, N = 752 (Bianconi et al., 2004), altitude de aproximadamente 400 m; MD- Morro do Diabo - SP, N = 345 (Reis et al. 1996), altitude de 600 m; BH- Município de Belo Horizonte - MG, N = 316 (Knegt et al. 2005); CV- Cinturão Verde de Cianorte - PR, N = 438, altitude de $530 \mathrm{~m}$ (Ortêncio Filho et al. 2005); JFMunicípio de Juiz de Fora, MG, N = 739 (Barros et al. 2006) complementado com dados do autor, altitude aproximada de 700 m; RT- Reserva Biológica do Tinguá - RJ, N = 655, altitude entre 65-1.270 m (Dias \& Peracchi 2008); RP- Município de Rio Preto - MG, N = 104 (Nobre et al. 1998) com complementação de dados pelo autor, altitude aproximada de 450 m; IT- Município de Itabira-MG, N = 180, altitude de 650-1.100 m (Silva et al., 2005); SA-Serra das Almas - CE, $N=180$, altitude de 750 m (Silva et al. 2004); SN- Serra Negra - MG, $\mathrm{N}=246$, altitude aproximada de $950 \mathrm{~m}$ (Moratelli \& Peracchi, 2007); SO- Parque Nacional da Serra dos Órgãos - RJ, N = 203, altitude entre 500-1.000 m; IB- Parque Estadual do Ibitipoca - MG, N = 213, altitude entre 900-1.400 m (não publicado) e SC-Serra do Caraça - MG, N = 245, altitude de 850-2.070 m (Falcão et al. 2003).

Figure 1. Dendogram obtained using UPGMA method and Euclidean distance for 13 surveys of Chiroptera including the region of Serra Negra and the following localities and states: FE- Fênix city - PR, N = 752 (Bianconi et al., 2004), approximate height 400 m; MD- Morro do Diabo - SP, N = 345 (Reis et al. 1996), height of 600 m; BH- Belo Horizonte city - MG, N = 316 (Knegt et al. 2005); CV- Cinturão Verde de Cianorte - PR, N = 438, height of $530 \mathrm{~m}$ (Ortêncio Filho et al. 2005); JF- Juiz de Fora city, MG, N = 739 (Barros et al. 2006) completed with author's informations, approximate height 700 m; RT- Biological Reserve of Tinguá - RJ, N = 655, height 65-1,270 m (Dias \& Peracchi 2008); RP- Rio Preto city - MG, N = 104 (Nobre et al. 1998), completed with author's informations, approximate height $450 \mathrm{~m}$; IT- Itabira city - MG, $\mathrm{N}=180$, height of 650-1,100 m (Silva et al., 2005); SA- Serra das Almas - CE, N = 180, height of 750 m (Silva et al. 2004); SN- Serra Negra - MG, N = 246, approximate height $950 \mathrm{~m}$ (Moratelli \& Peracchi, 2007); SO- National Park of Serra dos Órgãos - RJ, N = 203, height of 500-1,000 m; IB- State Park of Ibitipoca - MG, N = 213, height of 900-1,400 m (unpublished) ) e SC-Serra do Caraça - MG, N = 245, height of 850-2,070 m (Falcão et al. 2003).

da altitude, presença de cavernas e proximidade entre as duas áreas, o Parque Estadual do Ibitipoca e a Serra Negra compartilham de uma fitofisionomia muito semelhante, onde pode-se observar florestas nebulares entremeadas aos campos rupestres formando um mosaico vegetacional. Estas características comuns entre a Serra do Ibitipoca e Serra Negra, associadas à proximidade entre as duas localidades, influenciam nesta hipótese de agrupamento. A partir destas duas observações, podemos constatar que a Serra Negra sofre influência dos dois ambientes, podendo ser considerada uma área de transição com importantes implicações conservacionistas.

A abundância de S. lilium no Parque Estadual do Ibitipoca e na Serra Negra pode estar relacionada também à grande diversidade de espécies de Solanaceae, visto que Sturnira possui preferência por frutos do gênero Solanum (Zortéa, 2007). Em levantamentos 
florísticos na Mata Atlântica, a família Solanaceae se destaca entre as mais ricas em espécies (Barroso, 1957; Oliveira-Filho \& Fontes, 2000) e na Serra Negra, Feliciano (2008) registrou 19 espécies de Solanum, sendo principalmente encontradas no interior e borda de mata e nos campos rupestres. Esta autora, estudando a similaridade florística das Solanaceae da Serra Negra encontrou em suas análises valores altos de similaridade com o Parque Estadual do Ibitipoca, refletido por espécies de ampla distribuição geográfica.

Mello (2008), estudando a interação entre Solanaceae e S. lilium em região montanhosa, mostra que $S$. lilium é um eficiente dispersor de Solanaceae nesta área. Na Serra Negra, as Solanaceae são mais abundantes em altitudes que variam de 875 a $1.100 \mathrm{~m}$ (Feliciano, 2008), onde ocorreu também o maior volume de capturas de $S$. lilium. Através destas evidências, podemos sugerir que a presença abundante de S. lilium em altitudes superiores a $800 \mathrm{~m}$, deve estar relacionada à maior representatividade de espécies de Solanaceae nesta cota altitudinal que $S$. lilium acompanha a distribuição, diversidade e abundância das espécies de Solanum nestes ambientes. Em capturas realizadas no município de Rio Preto, localizado na base da Serra Negra e com altitude aproximada de $450 \mathrm{~m}$, as espécies mais abundantes foram A. lituratus e $P$. lineatus, reforçando a hipótese de que $S$. lilium é abundante em altitudes elevadas.

Apesar da existência de informações em forma de inventários para várias localidades somadas aos dados obtidos apresentados no atual estudo possibilitarem a realização de análise de agrupamento, a interpretação dos resultados deve ser feita com cautela. Deve-se considerar que não existe uma padronização na metodologia utilizada nos diversos levantamentos. Mesmo considerando as redes de neblina armadas no sub-bosque como o método adotado durante as capturas, variáveis como os locais de instalação das redes, datas escolhidas, fases da lua, podem influenciar no volume de capturas. O estado de conservação, fase de sucessão ecológica e dimensões dos fragmentos florestais estudados, oferta de recursos, presença de abrigos como cavernas e pequenas cavidades naturais em rochas, e atividade humana podem influenciar na riqueza de espécies das áreas amostradas.

Neste estudo foram registradas 15 espécies, uma riqueza de espécies relevante se considerado o número de 246 capturas. O levantamento se restringiu à vertente sul da região da Serra Negra que faz divisa com o estado do Rio de Janeiro sob forte influência climática da região serrana Fluminense. Contrapondo com esta área, a vertente norte da Serra Negra está voltada para o município de Lima Duarte, sul da Zona da Mata Mineira, próximo aos ambientes que formam o Parque Estadual do Ibitipoca e sul de Minas Gerais. Dessa forma as florestas da Serra Negra comportam-se como um ecótone com possibilidade de abrigar uma grande diversidade de morcegos, tornando esta região de grande importância para a conservação da biodiversidade na Zona da Mata de Minas Gerais.

\section{Agradecimentos}

À Fundação de Amparo a Pesquisa do Estado de Minas Gerais - FAPEMIG, pelo apoio financeiro ao desenvolvimento do projeto; ao Instituto Brasileiro do Meio Ambiente e Recursos Naturais Renováveis (IBAMA), pela concessão da licença para coleta e captura dos morcegos, ao Instituto Ambiental Vale do Rio Preto - IAVARP pelo apoio às atividades de campo na Serra Negra e à Universidade Federal de Juiz de Fora, pelo apoio e transporte ao campo. A Marco Antônio Manhães (Universidade Federal de Juiz de Fora) pela revisão e sugestões para o desenvolvimento do trabalho.

\section{Referências Bibliográficas}

BARROS, R.S., BISAGGIO, E.L., \& BORGES, R.C. 2006. Morcegos (Mammalia, Chiroptera) em fragmentos florestais urbanos no Município de Juiz de Fora, Minas Gerais, Sudeste do Brasil. Biota Neotrop. 6(1):http://www.biotaneotropica.org.br/v6n1/pt/abstract?inventory+bn 02206012006 (último acesso em 22/09/2009).

BARROSO, G.M. 1957. Solanaceae. In Flora do Itatiaia I. Rodriguésia. 20(32):75-88.

BARQUEZ, R.M., GIANNINI, NP. \& MARES, MA. 1993. Guide to the Bats of Argentina. Oklahoma Museum of Natural History, Norman, 119 p. (Publicações especias, n. VIII).

BENITES, V.M., CAIAFA, A.N., MENDONÇA, E.S., SCHAEFER, C.E. \& KER, J.C. 2003. Solos e vegetação nos complexos rupestres de altitude da Mantiqueira e do Espinhaço. Floresta Ambient. 10(1):76-85.

BIANCONI, G.V., MIKICH, A.B. \& PEDRO, W.A. 2004. Diversidade de morcegos (Mammalia, Chiroptera) em remanescentes florestais do município de Fênix, Noroeste do Paraná, Brasil. Rev. Bras. Zool. 21(4):943-954.

COSTA, C. \& HERRMANN, G. 2006. Plano de ação do corredor ecológico da Mantiqueira. 1 ed. Valor Natural, Belo Horizonte, 64 p.

DIAS, D. \& PERACCHI, A.L. 2008. Quirópteros da Reserva Biológica do Tinguá, Estado do Rio de Janeiro, sudeste do Brasil (Mammalia: Chiroptera). Rev. Bras. Zool. 25(2):333-369.

DRUMMOND, G.M., MARTINS, C.S., MACHADO, A.B.M., SEBAIO, F.A., ANTONINI, Y. 2005. Biodiversidade em Minas Gerais: um atlas para sua conservação. 2 ed. Fundação Biodiversitas, Belo Horizonte, 222 p.

ESBÉRARD, C.E.L. \& BERGALLO, H.G. 2003. A importância dos inventários de morcegos no Sudeste do Brasil. In Resumos do 2 Congresso Brasileiro de Mastozoologia. PUC Minas, Belo Horizonte, p. 70-71.

FALCÃO, F.C., REBELO, V.F. \& TALAMONI, S.A. 2003. Structure of a bat assemblage (Mammalia, Chiroptera) in Serra do Caraça Reserve, South-east Brazil. Rev. Bras. Zool. 20(2):347-350.

FELICIANO, A.F. 2008. Solanaceae A. Juss. da Serra Negra, Rio Preto, Minas Gerais. Dissertação de Mestrado, Universidade Federal de Juiz de Fora, Juiz de Fora, 135 p.

FELTON, M.B., ACHARYA, L. \& AUDET, D. 1992. Phyllostomid bats (Chiroptera: Phyllostomidae) as indicators of habitat disruption in the Neotropics. Biotropica. 24(3):440-446.

HEILBRON, M., TUPINAMBÁ, M., EIRADO, L.G., RIBEIRO, A., PACIULLO, F.V.P., TROUW, R.A., VALERIANO, C.M., JUNHO, M.C.B., ROIG, H.L., NOGUEIRA, J.R., MEDEIROS, R.M., ROCHA, D., POLONIA, J., SILVA, R.R. \& TOLEDO, C. 2000. Geologia das folhas Santa Rita do Jacutinga e Rio Preto. COMIG; UFMG; UFRJ; UERJ, Rio de Janeiro. (relatório técnico).

KNEGT, L.V., SILVA, J.A., MOREIRA, E.C. \& SALES, G.L. 2005. Morcegos capturados no município de Belo Horizonte, 1999-2003. Arq. Bras. Med. Vet. Zootec. 57(5):576-583.

MEDELLÍN, R.A., ARITA, H.T. \& SÁNCHEZ, H.O. 1997. Identificación de Los Murciélagos de México, Clave de campo. Asociación Mexicana de Mastozoología, Ciudade Universitaria, México, 83 p.

MELLO, M.A.R., KALKO, E.K.V. \& SILVA, W.R. 2008. Diet and abundante of the bat Sturnira lilium (Chiroptera) in a Brazilian Montane Atlantic Forest. J. Mammal. 89(2):485-492.

MITTERMEIER, R.A., GIL, P.R., HOFFMANN, M., PILGRIM, J., BROOKS, T., MITTERMEIER, C.G., LAMOUREX, J. \& FONSECA, G.A.B. 2005. Hotspots revisited: earth's biologically richest and most endangered terrestrial ecorregions. CEMEX; Agrupación Sierra Madre, Washington.

MORATELLI, R. \& PERACCHI, A.L. 2007. Morcegos (Mammalia, Chiroptera) do Parque Nacional da Serra dos Órgãos. In Ciência e Conservação da Serra dos Órgãos (C. Cronemberger \& E.B. Viveiros de Castro, orgs.). IBAMA, Brasília.

NOBRE, P.H., SALIMENA-PIRES, F.R., ESBÉRARD, C.E.L. \& SOUZA, B.M. 1998. Regeneração natural de florestas através da dispersão de sementes por morcegos na área do AHE de Mello, Rio Preto, MG. Principia. 3:113-122. 
OLIVEIRA-FILHO, A.T. \& FONTES, M.A.L. 2000. Patterns of floristic differentiation among Atlantic forests in south-eastern Brazil, and the influence of climate. Biotropica. 32:793-810.

ORTÊNCIO-FILHO, H., REIS, N.R., PINTO, D., ANDERSON, R., TESTA, D.A. \& MARQUES, M.A. 2005. Levantamento dos morcegos (Chiroptera, Mammalia) do Parque Municipal do Cinturão Verde de Cianorte, Paraná, Brasil. Chiropt. Neotrop. 11(1/2):211-215.

PERACCHI, A.L., LIMA, I.P., REIS, N.R., NOGUEIRA, M.R. \& ORTÊNCIOFILHO, H. 2006. Ordem chiroptera. In Morcegos do Brasil (A.L. Reis, A. L. Peracchi, W.A. Pedro \& I.P. Lima, eds.). Editora da Universidade Estadual de Londrina, Londrina, p. 153-219.

REDONDO, R.A.F., BRINA, L.P.S., SILVA, R.F., DITCHFIELD, A.D. \& SANTOS, F.R. 2008. Molecular systematics of the genus Artibeus (Chiroptera: Phyllostomidae). Molec. Phylog. Evolut. 49(1):44-58.

REIS, N.R., PERACCHI, A.L., MULLER, M.F., BASTOS, E.A. \& SOARES, E.S. 1996. Quirópteros do Parque Estadual Morro do Diabo, São Paulo, Brasil (Mammalia, Chiroptera). Rev. Bras. Biol. 56(1):87-92.

REIS, N.R., SHIBATTA, O.A., PERACCHI, A.L., PEDRO, W.A. \& LIMA, I.P 2006. Sobre os mamíferos do Brasil. In Morcegos do Brasil (A.L. Reis, A.L. Peracchi, W.A. Pedro \& I.P. Lima, eds.). Editora da Universidade Estadual de Londrina, Londrina, p. 7-26 PEDRO, W.A., LIMA, I.P., REIS, A.L. \& PERACCHI, A.L. mamíferos do Brasil. Editora da Universidade Estadual de Londrina, Londrina, $437 \mathrm{p}$.

REIS, N.R., SHIBATTA, O.A., PERACCHI, A.L., PEDRO, W.A. \& LIMA, I.P 2007a. Sobre os morcegos brasileiros. In Morcegos do Brasil (A.L. Reis, A.L. Peracchi, W.A. Pedro \& I.P. Lima, eds.). Editora da Universidade Estadual de Londrina, Londrina, p. 17-25.

REIS, A.L., PERACCHI, A.L. PEDRO, W.A. \& LIMA, I.P. 2007b. Morcegos do Brasil. Editora da Universidade Estadual de Londrina, Londrina, $253 \mathrm{p}$.
ROCHA, C.F.D., BERGALLO, H.G., ALVES, M.A.S. \& SLUYS, M.V. 2003. A biodiversidade nos grandes remanescentes florestais do estado do Rio de Janeiro e nas restingas da Mata Atlântica. Rima, São Carlos, 160 p.

SILVA, S.S.P., GUEDES, P.G., CAMARDELLA, A.R. \& PERACCHI, A.L. 2004. Survey of bats (Mammalia, Chiroptera) with coments on reproduction status, in Serra das Almas Private Heritage Reserve, in the state of Ceará, Northwestern of Brasil. Chiropt. Neotrop. 10(1/2):191-195.

SILVA, R., PERINI, F.A. \& OLIVEIRA, W.R. 2005. Bats from the city of Itabira, Minas Gerais, Southeastern Brazil. Chiropt. Neotrop. 11(1/2):216-219.

SIMMONS, N.B. \& VOSS, RS. 1998. The mammals of Paracou, French Guiana: a neotropical lowdland rainforest fauna. Bulletin of the American Museum of Natural History, New York, 219 p. (v. 237, parte 1, Bats).

STRAUBE, F.C., BIANCONI, G.V. 2002. Sobre a grandeza e a unidade utilizada para estimar esforço de captura com utilização de redes-deneblina. Chiropt. Neotrop. 8(1/2):150-152.

VALENTE, A.S.M. 2007. Composição, estrutura e similaridade florística do estrato arbóreo de três fragmentos de floresta atlântica, na Serra Negra, município de Rio Preto, Minas Gerais, Brasil. Dissertação de Mestrado, Universidade Federal de Juiz de Fora, Juiz de Fora, 69 p.

VELOSO, H.P., RANGEL-FILHO, A.L.R. \& LIMA, J.C.A. 1991. Classificação da vegetação brasileira adaptada a um sistema universal. IBGE, Rio de Janeiro.

VIZOTTO, L.D. \& TADDEI, V.A. 1973. Chave para determinação de quirópteros brasileiros. Gráfica Francal, São José do Rio Preto, 72 p.

ZORTÉA, M. 2007. Subfamília Sternodermatinae. In Morcegos do Brasil. (N.R. Reis, A.L. Peracchi, W.A. Pedro \& I.P. Lima, eds.). Editora da Universidade Estadual de Londrina, Londrina, p. 107-128.

Recebido em 26/04/09

Versão reformulada em 14/07/09

Publicado em 03/09/09 
Apêndice 1. Espécimes coletados e tombados como material testemunho na coleção de Chiroptera da Universidade Federal de Juiz de Fora - UFJF.

Apenddix 1. Collected and registered specimen as witness material in the Chiroptera collection of Universidade Federal de Juiz de Fora - UFJF.

Anoura caudifer UFJF-59; Carollia perspicillata UFJF-86, UFJF-92, UFJF-97; Desmodus rotundus UFJF-52, UFJF-190; Chrotopterus auritus UFJF-58, UFJF-202; Mimon bennettii UFJF-187; Micronycteris minuta UFJF-186; Artibeus lituratus UFJF-74, UFJF-98, UFJF-201; Artibeus fimbriatus UFJF-57, UFJF-189; Platyrrhinus lineatus UFJF-75, UFJF-76, UFJF-93; Pygoderma bilabiatum UFJF-53; Sturnira lilium UFJF-56, UFJF-188, UFJF-204; Vampyressa pusilla UFJF-54, UFJF-99; Eptesicus furinalis UFJF-197, UFJF-198; Myotis nigricans UFJF-55, UFJF-95; Myotis levisUFJF-195.

Apêndice 2. Tabela (matriz) de dados relacionando os inventários utilizados para comparações e análise de agrupamento. As siglas das localidades seguem aquelas apresentadas na legenda da Figura 1.

Apenddix 2. Tabel (matrix) of data relating the inventory used to comparation and analysis of group. The location's abreviatures follow the ones presented in the title of Figure 1.

\begin{tabular}{|c|c|c|c|c|c|c|c|c|c|c|c|c|c|}
\hline $\begin{array}{c}\text { Espécies / Localidades } \\
\mathbf{N}^{\circ} \text { de capturas }\end{array}$ & BH & IT & $\mathbf{S A}$ & SC & FE & $\mathbf{C V}$ & JF & MD & $\mathbf{S N}$ & SO & RT & IB & $\mathbf{R P}$ \\
\hline Anoura caudifer (E. Geoffroy, 1818) & 1 & - & - & 5 & - & - & 29 & - & 3 & 9 & 31 & 1 & 7 \\
\hline Anoura geoffroyi Gray, 1838 & - & 3 & 13 & 6 & - & - & - & - & - & 5 & 6 & - & - \\
\hline Artibeus cinereus (Gervais, 1856) & - & - & - & - & - & - & - & - & - & - & 5 & - & - \\
\hline Artibeus fimbriatus Gray, 1838 & - & - & 1 & - & 65 & 30 & 10 & - & 2 & 12 & 75 & - & 11 \\
\hline Artibeus planirostris Spix, 1823 & - & - & - & - & 46 & - & - & 19 & - & - & - & - & - \\
\hline Artibeus lituratus (Olfers, 1818) & 174 & 102 & 4 & 9 & 413 & 269 & 279 & 143 & 39 & 28 & 55 & 5 & 31 \\
\hline Artibeus obscurus (Schinz, 1821) & - & - & - & - & - & - & - & 7 & - & - & 31 & - & - \\
\hline Artibeus planirostris Spix, 1823 & - & 4 & - & - & - & - & - & - & - & - & - & - & - \\
\hline Carollia perspicillata (Linnaeus, 1758) & 3 & 5 & 71 & 7 & 148 & 35 & 89 & 33 & 52 & 44 & 176 & 7 & 8 \\
\hline Chiroderma villosum Peters, 1860 & - & - & - & - & 1 & - & - & - & - & - & - & - & - \\
\hline Chrotopterus auritus (Peters, 1856) & - & - & - & - & 3 & 2 & - & - & 3 & - & - & - & - \\
\hline Cynomops planirostris (Peters 1866) & - & - & 13 & - & - & - & - & - & - & - & - & - & - \\
\hline Desmodus rotundus (E. Geoffroy, 1810) & - & - & 4 & 8 & 5 & - & 62 & - & 47 & 5 & 15 & 98 & - \\
\hline Diphylla ecaudata Spix, 1823 & - & - & - & - & - & - & - & - & - & - & 1 & 1 & - \\
\hline Eptesicus brasiliensis (Desmarest, 1819) & - & 1 & - & 2 & - & - & - & - & - & - & 2 & - & - \\
\hline Eptesicus diminutus Osgood, 1915 & - & - & - & - & - & - & - & 2 & - & - & - & - & - \\
\hline Eptessicus furinalis (d'Orbigny, 1847) & - & - & - & - & - & - & 4 & - & 3 & - & - & - & - \\
\hline Eumops perotis (Schinz, 1821) & - & - & - & 2 & - & - & - & - & - & - & - & - & - \\
\hline Glossophaga soricina (Pallas, 1766) & 32 & 13 & 9 & - & - & - & 76 & 2 & - & - & 3 & - & - \\
\hline Histiotus velatus (I. Geoffroy, 1824) & - & - & - & - & - & - & 1 & - & - & - & - & - & - \\
\hline Lasiurus blossevillii (Lesson y Gamot, 1826) & - & - & - & 2 & 2 & - & - & - & - & - & - & - & - \\
\hline Lasiurus ega (Gervais, 1856) & - & 2 & - & - & - & - & - & - & - & - & - & - & - \\
\hline Lasiurus cinereus (Palisot de Beauvois, 1796) & - & - & - & - & - & 4 & - & - & - & - & - & - & - \\
\hline Lonchophylla bokermanni Sazima, Vizotto \& Taddei, 1978 & - & - & - & - & - & - & - & - & - & 1 & 10 & - & - \\
\hline Micronycteris microtis Miller, 1898 & - & - & - & - & - & - & - & - & - & - & 3 & - & - \\
\hline Micronycteris megalotis (Gray, 1842) & - & - & - & - & 1 & - & - & - & - & 7 & - & 1 & - \\
\hline Micronycteris minuta (Gervais, 1856) & - & - & - & - & - & - & - & - & 1 & - & - & - & - \\
\hline Mimon bennettii (Gray, 1838) & - & - & - & - & - & - & 1 & - & 1 & - & 1 & - & - \\
\hline Molossops temminckii (Burmeister, 1854) & - & - & - & - & - & - & - & 4 & - & - & - & - & - \\
\hline Molossus molossus (Pallas, 1766) & - & 2 & - & 3 & - & 1 & 19 & - & - & 3 & 85 & - & 1 \\
\hline Molossus rufus E. Geoffroy, 1905 & - & - & - & - & - & 20 & - & - & - & - & 12 & - & - \\
\hline Myotis levis (I. Geoffroy, 1824) & - & - & - & - & 2 & - & - & - & 1 & 4 & 6 & - & - \\
\hline Myotis nigricans (Schinz, 1821) & 1 & 2 & - & 21 & 13 & 6 & 22 & 19 & 2 & 10 & 8 & 2 & 3 \\
\hline Myotis ruber (E. Geoffroy, 1806) & - & - & - & - & - & - & - & - & - & 9 & 2 & - & - \\
\hline Noctilio leporinus (Linnaeus, 1758) & - & - & - & - & - & - & - & 1 & - & - & - & - & - \\
\hline Noctilio albiventris Desmarest, 1818 & - & - & - & - & - & - & - & 8 & - & - & - & - & - \\
\hline Myotis riparius Handley, 1960 & - & - & - & - & 1 & - & - & - & - & - & 7 & - & - \\
\hline Nyctinomops macrotis (Gray, 1840) & - & - & - & - & - & - & 1 & - & - & - & - & - & - \\
\hline Peropteryx macrotis (Wagner, 1843) & - & - & - & - & - & - & - & - & - & - & 3 & - & - \\
\hline Phyllostomus hastatus (Pallas, 1767) & - & 1 & 2 & - & - & - & - & 4 & - & - & - & - & - \\
\hline Phyllostomus discolor Wagner, 1843 & 13 & - & 25 & - & - & - & - & - & - & - & - & - & - \\
\hline Platyrrhinus recifinus (Thomas, 1901) & - & - & - & - & - & - & - & 4 & - & 4 & 18 & - & - \\
\hline Platyrrhinus lineatus (E. Geoffroy, 1810) & 77 & 23 & 1 & 10 & - & 3 & 63 & - & 6 & - & 1 & - & 28 \\
\hline Pteronotus parnelli (Gray, 1843) & - & - & 10 & - & - & - & - & - & - & - & - & - & - \\
\hline Pygoderma bilabiatum (Wagner, 1843) & - & 4 & - & 7 & - & 1 & 9 & 10 & 1 & 2 & 10 & - & 1 \\
\hline Saccopterix leptura (Schreber, 1774) & - & - & - & - & - & - & - & - & - & - & 2 & - & - \\
\hline Sturnira lilium (E. Geoffroy, 1810) & 15 & 18 & 14 & 159 & 50 & 65 & 74 & 88 & 83 & 57 & 75 & 103 & 7 \\
\hline Tadarida brasilliensis (I. Geoffroy, 1824) & - & - & - & 4 & - & - & - & - & - & - & - & - & - \\
\hline Tonatia bidens (Spix, 1823) & - & - & 1 & - & - & - & - & - & - & - & 4 & - & - \\
\hline Trachops cirrhosus (Spix, 1823) & - & - & 2 & - & - & - & - & - & - & - & - & - & - \\
\hline Uroderma bilobatum Peters, 1866 & - & - & - & - & - & 2 & - & - & - & - & - & - & - \\
\hline Vampiressa pusilla (Wagner, 1843) & - & - & - & 1 & 2 & 2 & - & 1 & 3 & - & 8 & - & 7 \\
\hline
\end{tabular}

